\title{
Hyaluronan: its potential application in intervertebral disc regeneration
}

\author{
This article was published in the following Dove Press journal: \\ Orthopedic Research and Reviews \\ 23 March 2010 \\ Number of times this article has been viewed
}

\section{Bojiang Shen \\ Aiqun Wei \\ Divya Bhargav \\ Thomas Kishen \\ Ashish D Diwan}

Orthopaedic Research Institute, Department of Orthopaedic Surgery, St. George Hospital, The University of New South Wales, Sydney, Australia
Correspondence: Bojiang Shen

Orthopaedic Research Institute, Department of Orthopaedic Surgery, St. George Hospital, The University of New South Wales, 4-10 South Street, Kogarah, NSW 2217, Australia

$\mathrm{Tel}+6|29| 132248$

Fax +6I 291133967

Email b.shen@unsw.edu.au
Abstract: Hyaluronan (HA) is a ubiquitous component of extracellular matrix in human tissues with diverse functions in skeletal biology. The biophysical properties of HA, such as high viscosity, elasticity and highly negative charge, make it useful in various therapeutic procedures. Although intra-articular administration of HA has been extensively used in the management of osteoarthritis (OA), there is a paucity of data on the clinical application of HA in intervertebral disc repair. This review discusses the biology and signaling mechanisms of HA, the pathophysiology of disc degeneration and summarises current evidence relating to the role of HA in cell phenotype maintenance, differentiation of chondrocytes, intervertebral disc cells and bone marrow stromal cells, and its application in tissue engineering. Based on recent advances in the clinical outcomes of OA treatment, HA has demonstrated potential as a bio-polymer filler, therapeutic agent and cell carrier in the management of intervertebral disc degeneration.

Keywords: hyaluronan, cartilage, intervertebral disc, stromal cells, tissue engineering, back pain

\section{Introduction}

The successful regeneration of cartilage and intervertebral disc (IVD) requires reparative cells with corresponding signaling molecules and a microenvironment that supports the proliferation and differentiation of those cells. Biomaterials which mimic the natural microenvironment may play a key role in tissue-engineered repair of damaged and degenerated tissue.

Hyaluronan (HA), also known as hyaluronic acid, is a ubiquitous component of the extracellular matrix in human tissue. High concentrations of HA have been detected in skin, cartilage, nucleus pulposus of IVD, vitreous body of eye and synovial fluid. Initially isolated from the vitreous body by Meyer and Palmer in 1934, ${ }^{1} \mathrm{HA}$ has also been purified with varying molecular mass from joint fluid, umbilical cord, rooster comb and certain strains of streptococci. ${ }^{2}$ Although the majority of commercially available HA is prepared from rooster comb, HA production by fermentation of streptococci has the advantage of low cost, easy purification and higher molecular mass compared to traditional tissue extraction methods. ${ }^{3}$ The biophysical properties of HA such as high viscosity, elasticity and highly negative charge make HA useful in various therapeutic procedures. HA has been widely used in the cosmetic, nutritional, pharmaceutical and medical fields. The clinical application of HA has been classified as viscosurgery (in ophthalmic surgery to maintain a deep anterior chamber, reduce corneal trauma and push back the vitreous face), viscoaugmentation (to fill facial wrinkles, depressed scars and for cosmetic corrections), viscoseparation (to prevent post-operative adhesions), viscoprotection (to promote healing of wounded or injured tissue) and 
viscosupplementation (intra-articular administration for the treatment of osteoarthritis). ${ }^{4-6}$

Recent research has recognized the role of HA beyond its "bio-lubricant" and "bio-polymer filler" functions. HA plays an important role in embryonic development, tissue organization, wound healing, angiogenesis, cell migration, pericellular matrix formation and tumor invasion. ${ }^{7-9} \mathrm{HA}$ also possesses anti-inflammatory activity and is under evaluation to be used as a therapeutic agent for a number of inflammatory disorders. ${ }^{10}$ In addition, HA-derived gel has also shown anti-adhesive effect following knee surgery in a rabbit model. ${ }^{11}$ The combination of anti-inflammatory, anti-fibrotic and anti-adhesive activities of HA suggests its potential application in the prevention of post-operative adhesions and promotion of tissue repair.

The unique biophysical characteristics, biocompatibility, non-immunogenic properties and ease of size chain manipulation make HA a useful biomaterial for scaffold and carrier design in tissue engineering. This article aims to review recent advances relating to the application of HA in cartilage regeneration and tissue engineering with specific reference to IVD regeneration.

\section{Hyaluronan - the molecule}

HA is a linear high-molecular-mass non-sulfated glycosaminoglycan (GAG) composed of repeating disaccharide units of $\beta$-1, 4-glucuronic acid and $\beta$-1, 3-N-acetyl-D-glucosamine (Figure 1). It is the only GAG that is not synthesized in the Golgi apparatus and its synthesis does not require attachment to a core protein. In eukaryotes, HA is synthesized on the inner surface of the plasma membrane as an unadorned linear polymer. This polymerizing process is catalyzed by membrane-bound HA synthases (HAS) using UDP-sugar nucleotide precursors as substrates. There are three HAS isoforms designated as HAS1, HAS2 and HAS3, encoded by three related HAS genes at a different chromosome location. Concomitant with elongation of the HA polymer, it is extruded through the membrane into the extracellular matrix. HA exists in the bound and free status in the body with a very rapid turnover rate. HA catabolism is achieved either by hyaluronidase-mediated enzymatic degradation or by chemical mechanisms to generate fragments of various sizes. ${ }^{12,13}$

Newly synthesized HA chains contain 2500 to 25,000 repeating units with a molecular mass of $10^{6}$ to $10^{7} \mathrm{Da}$. The HA molecule is readily soluble in water and behaves as a stiffened random coil with a large hydrated volume allowing the molecules to interact with each other to create viscoelastic solutions. HA, with its high molecular mass, forms a specific stable tertiary structure in aqueous solution equivalent to the $\beta$-sheets seen in proteins. ${ }^{14}$ The viscosity is the most distinctive property of HA in the hydrated state largely depends on the length of the chains or molecular mass. For example, the viscosity of a $1 \%$ HA solution (a molar mass of $3-4 \times 10^{6}$ $\mathrm{Da}$ ) is about 500,000 times that of water. The viscosity may vary with the shear rate and is positively related to molar mass and concentration. ${ }^{13}$ Nevertheless, high molecular weight $\left(\mathrm{MW}>17 \times 10^{6}\right)$ HA produces high viscosity solutions by extending the degree of entanglement which cannot be achieved simply by increasing the concentration of low molecular weight HA. ${ }^{15}$ The extreme aqueous solubility and high turnover rate of HA in the body limit its application in tissue engineering. While maintaining the viscoelasticity, the physio-chemical properties of HA have been improved by chemical crosslinking and modification such as esterification to reduce the solubility and increase its stability and biocompatibility.

HA has diverse functions in skeletal biology including joint cavity formation and longitudinal bone growth. HA plays a critical structural role as a backbone of proteoglycan aggregates with large amount of proteoglycan monomers anchoring on it through a link protein in the extracellular matrix of tissues including articular cartilage and IVD (Figure 2). The HA-immobilized proteoglycan aggregates have enormous

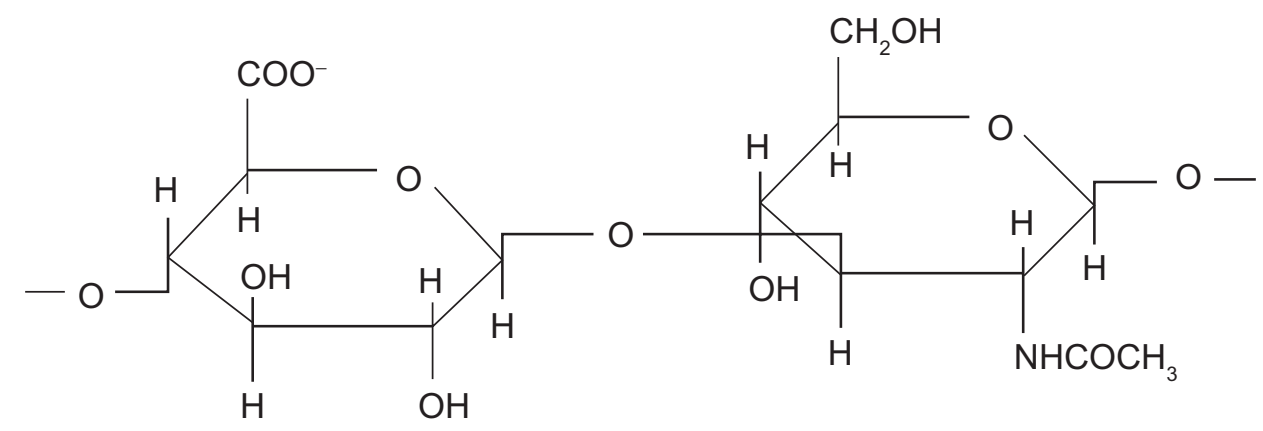

Figure I Structure of the repeating disaccharide unit of hyaluronan. 
molar masses and are embedded within a collagenous framework, limiting the opportunity for proteoglycan loss from tissue. ${ }^{13}$ HA synthesis and degradation are closely regulated in skeletal tissues and aberrant synthetic or degradative activity causes diseases. ${ }^{12}$ In addition, HA is structurally and chemically non-species-specific making it non-allergenic, unlike collagen.

\section{Interaction with cell-associated HA-binding proteins and signaling mechanism}

The HA-dependent functions of cells are triggered by an initial interaction with cell-associated HA-binding proteins or hyaladherin (ie, receptors) and subsequent activation of intracellular signaling cascades. So far, three major HA receptors have been identified: CD44, Receptor for HA mediated motility (RHAMM, also known as CD168) and intercellular adhesion molecule-1 (ICAM-1, also known as CD54). CD44, the main HA receptor, is extensively expressed as a group of multiple variant isoforms derived from extensive alternative splicing of mRNA and exists in inactive non-binding forms or an active ligand-binding form. Standard CD44 is the most abundantly expressed isoform. The binding of CD44 to HA plays an important role in cell adhesion to extracellular matrix components and is implicated in the stimulation of aggregation, proliferation, migration and angiogenesis. ${ }^{8,16}$ Other HA receptors, RHAMM and ICAM-1, are less well studied. RHAMM is localized inside the cell and released by certain, poorly defined stimuli. The interaction of HA with cell surface ICAM-1 is likely to have important implications for cell adhesion in normal and in disease states such as inflammation, atherosclerosis and cancer. ${ }^{17}$

The interaction of HA with cell surface receptors activates a variety of protein tyrosine and serine/threonine kinases including

A

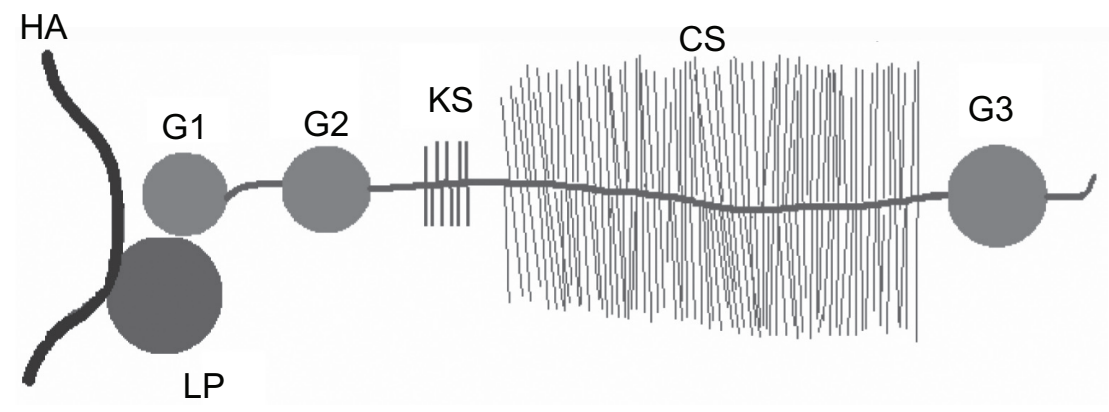

B

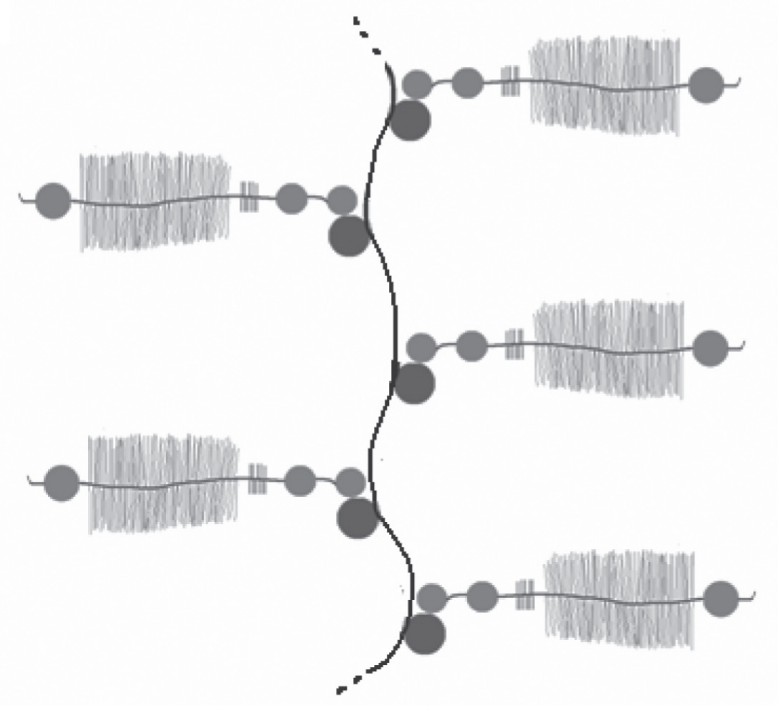

Figure 2 Schematic representation of hyaluronan (HA) backbone in aggregated proteoglycan structure. A) Proteoglycan monomer showing the GI, G2 and G3 globular domains. The N-terminal GI domain binds to HA through link protein (LP), and keratan sulfate (KS) and chondroitin sulfate (CS) attach to the core protein. B) Proteoglycan aggregate showing proteoglycan monomers anchoring on HA chain through link proteins. 
the non-receptor protein tyrosine kinase Src, HER2/Neu receptor, focal adhesion kinase, protein kinase $\mathrm{C}$ and MAP kinases. Interactions with specific HA receptors are believed to selectively couple with their specific downstream signaling pathways. As a consequence of regulating these kinases, HA promotes expression of specific cytokines and proteins involved in extracellular matrix remodeling. ${ }^{18} \mathrm{HA}$ counteracts the catabolic processes induced by interleukin-1 $\beta$ (IL-1 $\beta$ ) and block IL-1 $\beta$ stimulated the production of matrix metalloproteinase (MMP). The mechanism of this inhibition has been speculated to occur through HA binding to ICAM-1, leading to downregulation of nuclear factor

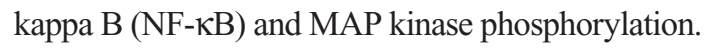

The signaling transduction mediated by degraded HA fragments through CD44 is associated with the post-injury inflammatory response, which demonstrates the opposite biological functions of native high molecular mass HA. The low molecular mass HA fragments function as potential endogenous "danger signals", while even smaller fragments can ameliorate these effects. ${ }^{19}$ Understanding the array of signaling pathways of inflammatory and anti-inflammatory effects of HA will encourage the development of novel antidegradation strategies when using HA in combination with other disease-modifying anti-arthritic drugs.

\section{Hyaluronan helps maintain chondrocyte phenotype}

Articular cartilage, a complex tissue capable of withstanding large compressive loads during daily activity, has poor regenerative capacity. Articular cartilage chondrocytes are highly specialized cells that produce extracellular matrix and express chondrogenic genes. Chondrocytes express the standard isoform of CD44 receptors and the CD44-HA interaction is necessary for organization, retention and regulation of normal cartilage matrix. Supra-molecular organization of HA molecules and the viscoelasticity in the hydrated state are responsible for some of the structural properties of cartilage. Immobilizing proteoglycan in the cartilage is another important function of HA.

Chondrocytes readily lose their phenotypic characteristics in a monolayer culture system while retaining their original characteristics in three-dimensional (3D) constructs. ${ }^{20}$ Addition of HA into collagen scaffolds seeded with articular chondrocytes improves the 3D environment and enhances chondrocyte growth, function and matrix deposition. ${ }^{21}$ Benzyl derivatives of HA (Hyalograft ${ }^{\circledR} \mathrm{C}$ and HYAFF-1 $1^{\circledR}$ ), are hydrophobic, biodegradable polymers which degrade at predictable rates and are compatible with in vitro cultured chondrocytes. Articular chondrocytes from osteoarthritic and healthy individuals cultured on HYAFF-11 for 3 weeks resulted in a significant increase in type II collagen, Sox 9 and aggrecan, and a reduction of type I collagen and downregulation of the catabolic MMPs compared to cells grown on culture plastic. The study revealed the effectiveness of HA in promoting cartilage matrix synthesis and maintaining the chondrocytic phenotype thereby providing an ideal construct for chondrocyte growth in vitro. ${ }^{22}$ Chondrocytes derived from cartilages of bovine, ${ }^{23}$ rabbits, ${ }^{24}$ rat $^{25}$ and swine ${ }^{26}$ encapsulated into HA-based scaffolds exhibited chondrocyte re-differentiation, enhancement of proteoglycan synthesis, increased expression of type II collagen and cartilage oligomeric matrix protein (COMP).

Exogenous HA can incorporate into cartilage and its beneficial effects on the proliferation, differentiation, migration, and phenotype maintenance of chondrocytes have been studied in vitro. Bovine chondrocytes grown in gelatin sponges with exogenous HA increase cellular retention and proteoglycan production. ${ }^{27}$ Rabbit chondrocytes embedded in collagen gels and cultured in a growth medium containing various concentrations of HA has shown enhancement of chondroitin sulfate synthesis and cellular proliferation at low concentration of HA. ${ }^{28}$ Bovine articular chondrocytes cultured in alginate constructs in a HA-containing medium led to significantly increased DNA, sulfated glycosaminoglycan and hydroxyproline synthesis. ${ }^{29}$

Besides the ability of HA to enhance cartilage matrix synthesis and chondrocyte growth, HA protects chondrocyte from apoptosis during the development of OA, playing a role in chondroprotection. ${ }^{30}$ Grishko et al exposed cultured human chondrocytes to reactive oxygen and mitochondriadriven apoptosis was induced with pro-inflammatory cytokines. Chondrocytes pre-treated with HA exhibited reduced mitochondrial DNA damage and enhanced mitochondrial DNA repair capacity and cell viability when compared to cells treated with only the cytokines. Addition of anti-CD44 antibody at saturating concentrations was found to abolish the protective effects of HA. The chondroprotective action of HA may be a consequence of preservation of mitochondrial function and amelioration of mitochondria-driven apoptosis, ${ }^{31}$ suggesting the role of HA in the management of OA other than through viscosupplementation.

\section{Hyaluronan promotes chondrogenic differentiation of bone marrow stromal cells}

Bone marrow multipotent mesenchymal stromal cells (BM MSCs) are capable of differentiating into various cell types 
including chondrocytes and are a potential source for stem cell-based IVD regeneration and repair. Lineage-directed differentiation of BM MSCs requires the appropriate cellular microenvironment including signal molecules and scaffold/carriers.

HA has been applied as either signal molecules or scaffold/carrier to induce MSC differentiation. HA, when supplemented in specific media, induces chondrogenesis of equine BM MSCs in a micromass pellet culture as evidenced by analysis of chondrocytic marker expression, such as proteoglycans and type II collagen. The chondrogenic efficacy of HA is pronounced, though lower than that of TGF- $\beta 1 .{ }^{32}$ A HA-based scaffold (a polymer derived from the total esterification of sodium hyaluronate) supported the adhesion, high viability, migration and proliferation of embedded BM MSCs, suggesting its potential in tissue repair. ${ }^{33}$ BM MSCs grown in a HA scaffold can undergo either chondrogenic or osteogenic differentiation depending on the presence of specific inducting factors in the culture media. ${ }^{34,35}$ Variations in the molecular weight of HA have a mild influence on the onset of chondrogenesis of MSCs in pellet culture, but do not significantly affect the outcome of the differentiation on day 21 culture. ${ }^{36}$ HA-based hydrogel particles have been used as a biomimetic growth factor delivery system to stimulate the chondrogenic differentiation of the cultured mesenchymal stem cells via the controlled release of bone morphogenetic protein-2 (BMP-2). ${ }^{37}$

The MSC seeded HA scaffolds over 3 weeks of culture produced a white, solid tissue with increased cell proliferation and type II collagen expression during this period of time, but could not achieve the same level of mechanical strength as human cartilage. ${ }^{38}$ To improve the mechanical and biological properties of HA scaffold, an array of HA-based bioactive composite scaffolds were designed to mimic the physiological environment present during tissue formation. A HA - silk fibroin composite scaffold, which combines the biological characteristics of HA with silk fibroin's superior mechanical properties, made BM MSCs undergo efficient differentiation and tissue formation measured by the specific gene expression analysis compared to plain silk fibroin scaffolds. ${ }^{39}$ A composite scaffold, prepared by homogeneously mixed $5 \%$ to $30 \%$ of gelatin with esterified HA, was found to be superior to pure HA scaffold for induction of chondrogenic differentiation of BM MSCs. ${ }^{40}$ BM MSCs in the composite scaffold, when implanted into a medial meniscus defect in a rabbit model, demonstrated chondrogenic differentiation and an ability to repair the tissue with meniscus-like fibrocartilage integrated with the host tissue. ${ }^{41}$ Utilization of autologous BM
MSCs in a HA scaffold for repair of an osteochondral defect in the rabbit knee has also shown promising results. ${ }^{42}$

The principal drawback of the majority of hydrogels is the difficulty in recovering cells from the $3 \mathrm{D}$ environment. Recently, Zhang et al used synthetic crosslinkers (disulfidecontaining polyethylene glycol diacrylate) to crosslink thiolmodified hyaluronan and gelatin macromonomers in the presence of cells. BM MSCs showed excellent viability and growth in the scaffold and could be readily liberated by dissociating the gel using the thiol-disulfide exchange reaction after cell expansion or differentiation. ${ }^{43}$

\section{The application of HA in tissue engineering}

HA has been manufactured in a variety of physical forms including hydrogels, sponges, meshes, fibres and fabrics that allow the development of a variety of HA-based scaffolds. HA, as a biomaterial, is biocompatible, non-toxic, non-immunogenic and non-inflammatory, making it ideal for the controlled release of drugs, DNA, peptides and other biomolecules. More importantly, HA can be used as cell carrier in tissue engineering and regenerative medicine. ${ }^{44,45}$

HA hydrogels has been used to deliver DNA into the cellular microenvironment. The carboxylate groups of HA can be created as a cross-linked hydrogel for DNA entrapment and also for drug delivery. HA hydrogels can maintain in vitro degradation rates and slow the release of drugs from hours to several days. ${ }^{46,47}$ Similarly, HA hydrogels and DNA-HA matrix have been used as a depot system for controlled gene or DNA delivery for tissue regeneration..$^{48}$ DNA immobilized to the substrate of crosslinked HA-collagen hydrogels can be slowly released and taken up by adherent cells demonstrated by expression of the transgene within these cells. ${ }^{49}$

HA-based scaffolds or nanogel systems have been investigated to target specific genes by delivery of siRNA therapeutics. Cellular uptake and gene silencing efficiency were achieved at much higher level in CD44 over-expressing cell lines than CD44 negative cell lines. Using HA conjugates with polycations to target intracellular delivery of siRNA by the receptor-mediated endocytosis significantly improved gene-silencing efficiency. Thus HA conjugated into siRNA complex has the potential to be utilized in the treatment of diseases in tissues with HA receptors..$^{50,51}$

In addition to application for biomolecules and drug delivery, HA based scaffolds have the potential to be used as a cell delivery system. ${ }^{52}$ Composite HA and type I collagen hydrogels have been used to deliver porcine chondrocytes into 3D designed poly (propylene fumarate) scaffolds to increase seeding effi- 
ciency and cellular retention. Chondrocytes were encapsulated into the hydrogels with varying concentrations of HA. The tissue-engineered scaffolds were implanted subcutaneously in immunocompromised mice for 4 weeks. The chondrocytes in scaffolds with 5\% HA/collagen hydrogel exhibited a significant increase in proteoglycan synthesis and larger numbers of chondrocytes retained their metabolic activity both in vivo and in vitro. The authors suggested that a combination of composite HA hydrogels and the designed scaffolds could provide a functional tissue-engineered construct for cartilage repair with enhanced tissue regeneration. Pereira et al developed a novel injectable carrageenan/fibrin/hyaluronic acid-based hydrogel with in situ gelling properties to be seeded with chondrogenic cells and used for cartilage tissue engineering applications. The in vitro cell culture study showed that the seeded human articular chondrocytes were able to proliferate and form cartilage specific extracellular matrix in the hydrogel. Further using an organ culture defect model, experimentally generated defects on bovine cartilage cubes were filled with the injectable hydrogel seeded with chondrocytes to form organ constructs. Regeneration of bovine articular cartilage was observed 6 weeks after the organ constructs were implanted subcutaneously into nude mice. ${ }^{53} \mathrm{~A}$ fibrin/HA composite gel has also been used as a cell delivery system to implant rabbit chondrocytes into nude mice and then into full thickness cartilage defects in a rabbit model. The implanted chondrocytes showed improved glycosaminoglycan synthesis and cartilage formation. ${ }^{54}$ Overall, these studies show the potential of HA-based scaffolds as a suitable delivery vehicle for chondrogenic cells in cartilage regeneration.

\section{The potential of HA in IVD regeneration}

The IVD between the vertebral bodies is an avascular and aneural structure composed of an outer fibrous annulus fibrosus (AF), an inner jelly-like nucleus pulposus (NP) and adjacent cartilaginous end-plates (EP) (Figure 3). Although accounting for only $1 \%$ of the disc volume, IVD cells sustain disc integrity by production and maintenance of the extracellular matrix, predominantly composed of proteoglycans, collagens, noncollagenous proteins and large amount of water molecules. The balance between synthesis and breakdown determines quality and integrity of the matrix and mechanical behavior of the disc. IVD degeneration is initiated by a progressive reduction of the extracellular matrix, caused by disc cell death, impaired cell function and accelerated matrix degradation. ${ }^{55}$

Although the multifactorial etiology is not clearly understood, IVD degeneration is closely associated with genetic factors, mechanical loading of the spine, loss of prospective potency of NP cells and alterations in the diffusion of nutrients into the disc. A variety of inflammatory mediators have been implicated in the IVD degeneration including nitric oxide (NO), interleukins, MMPs, prostaglandin E2 (PGE2), tumor necrosis factor- $\alpha$ (TNF- $\alpha$ ) and a group of cytokines. ${ }^{56}$ Similar to cartilage tissue, the disc lesions have poor intrinsic potential for spontaneous healing. The degeneration sets off a cascade that further affects other spinal structures and ultimately leads to extensive structural defects and loss of normal motion segment function. ${ }^{55}$

The role of HA in cell-based tissue engineering for disc repair has been studied. A gelatin/chondroitin-6-sulfate/HA tri-copolymer was developed to serve as a bioactive scaffold to help human NP cells preserve their viability, proliferation and to promote matrix synthesis. ${ }^{57}$ The HA-based scaffold enhanced the production of proteoglycan and type II collagen in the extracellular matrix and induced re-differentiation of human NP cells. A cross-linked collagen-HA scaffold maintained cell viability and proliferation of embedded bovine NP cells, retained a high proteoglycan synthesis rate and the low elution of sulfated glycosaminoglycan into the surrounding medium over a 7-day culture period. ${ }^{58}$ The function of bovine AF and NP cells was maintained even after culture in the collagen/HA scaffold for up to 60 days. ${ }^{59}$ HA may be a cell differentiation inducing factor intended for biological treatment strategies of the IVD. ${ }^{60}$ These studies exhibit the ability of HA in promoting extracellular matrix synthesis and cell viability in vitro.

Anti-inflammatory effects of intradiscally injected HA have been shown in animal models. Temporal changes in the proinflammatory cytokine and macrophage profiles have been detected in the epidural space of post-laminectomy disc treated with or without topical high molecular weight HA gel in a rat model. HA gel decreased the number of monocytes/ macrophages and the concentration of certain cytokines in the early inflammatory phase of healing, thereby showing potential to decrease inflammation, fibrosis and scar formation in the post-laminectomy rats. ${ }^{61}$ No significant difference was observed in pain related behavior in response to proliferative scar within the spinal canal between the sham and HA gel treatment groups in the post-laminectomy rat model. ${ }^{62}$

The therapeutic potential of HA as a cell carrier for disc regeneration has been explored in animal models. Human BM MSCs encapsulated in HA gel were embedded in a disc like construct and induced to undergo chondrogenesis in vitro. The cells exhibited a chondrocytic phonotype and maintained the microarchitecture of a native IVD. This 


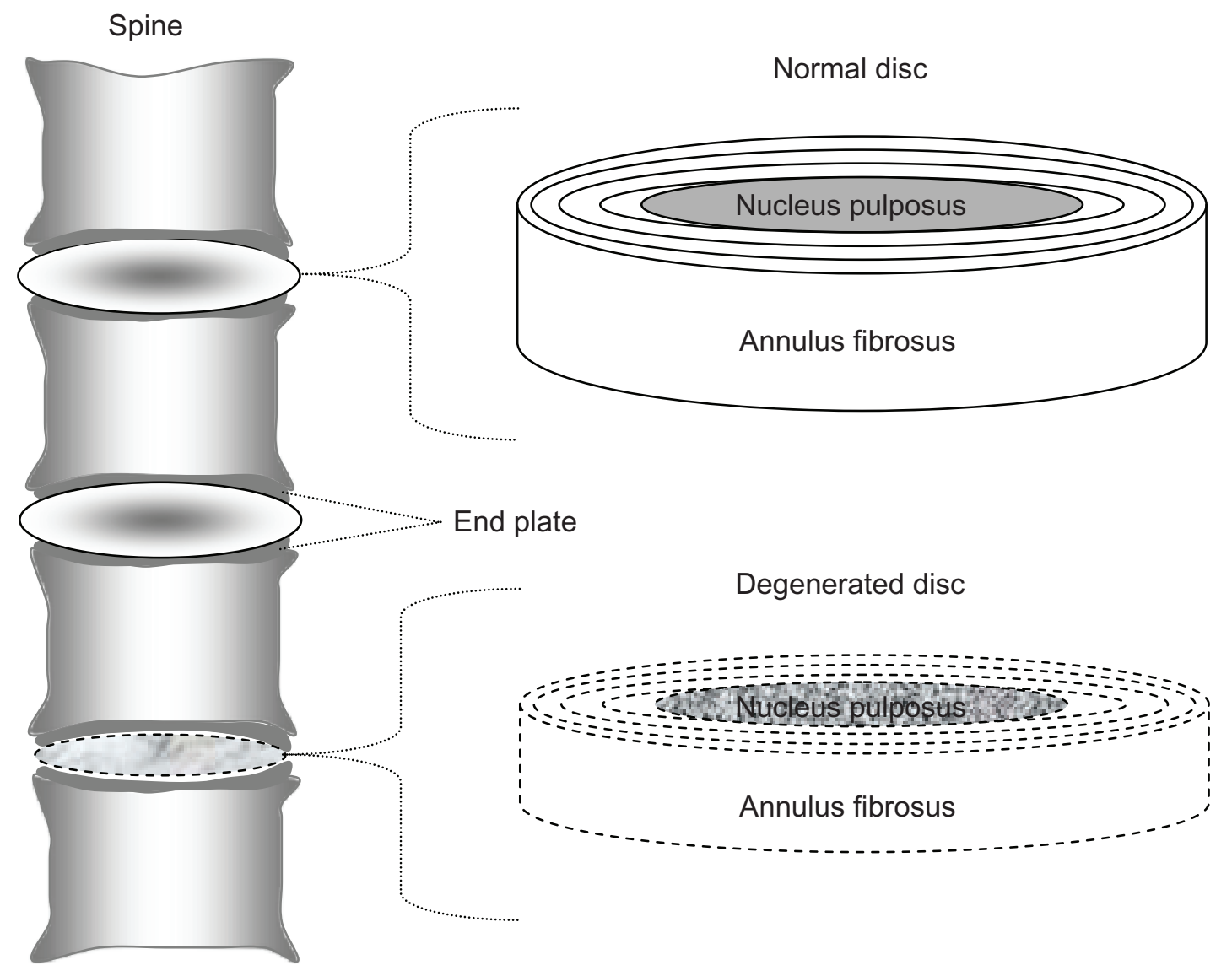

Figure $3 \mathrm{~A}$ diagram of the spinal segment consisting of vertebral bodies with intervertebral discs sandwiched between them. Compared to a normal disc, the degenerated disc undergoes morphological changes observed as reduced height and more disorganized structure due to loss of water and extracellular matrix.

MSC/HA-based disc construct demonstrates a potential for the tissue engineering of degenerated IVD. ${ }^{63} \mathrm{HA}$ gel has also been studied as a cell carrier to directly deliver BM MSCs into rat coccygeal discs. ${ }^{64}$ Revell et al examined the effects of two HA-derived biodegradable polymers, HYAFF $^{\circledR}-120$ and $\mathrm{HYADD}^{\circledR}-3$, on the repair of damaged IVD in a pig nucleotomy model. The autologous BM MSCs were embedded in the HA gel and then injected into the nucleotomized discs. The implanted HA with or without MSCs maintain the normal biconvex structure of NP and contained viable cells forming matrix at 6 weeks when compared to the control group undergoing nucleotomy only. ${ }^{65}$ The initial data suggest that HA has the potential for construction of an engineered IVD for the biological repair of degenerated discs.

\section{Clinical application of HA in the treatment of osteoarthritis}

The clinical application of HA for musculoskeletal disorders mainly focuses on the treatment of osteoarthritis
(OA), through a viscosupplementation (intra-articular administration of HA) procedure. OA is a degenerative condition affecting synovial joints to cause pain and reduced mobility. Macroscopic changes in an arthritic joint include articular cartilage destruction, osteophyte formation and breakdown of the osteochondral junction. At the molecular level, chondrocytes and synovial cells of OA patients produce increased levels of inflammatory cytokines, such as IL- $1 \beta$ and TNF- $\alpha .{ }^{66}$ IL- $1 \beta$ decreases synthesis of type II collagen, proteoglycans and increases synthesis of MMPs. In addition, a 33\% to 50\% reduction in the concentration and molecular weight of HA in osteoarthritic joints reduces the viscoelasticity of the synovial fluid. ${ }^{67}$

The enormous interest in this molecule is evidenced by vast amount of published literature and the large number of randomized controlled clinical trials (RCT). ${ }^{68}$ Intra-articular administration of HA increases the viscoelasticity and restores the lubricating and shock-absorbing properties of 
the synovial fluid. Clinical trial data have supported the intra-articular HA therapy as not only a symptom-modifying therapy but also a treatment which may significantly decrease the rate of deterioration of joint structure. ${ }^{6}$ HA has shown cartilage-protective and anti-inflammatory effects during progression of osteoarthritic degeneration by inhibiting the degenerative changes and enhancing the synthesis of proteoglycans and extracellular matrix proteins of chondrocytes. HA decreases nociceptive activity in inflamed joints ${ }^{69,70}$ and protects the cartilage by reducing reactive oxygen radicals, inflammatory mediators and matrix-degrading enzymes. HA has an analgesic effect when injected intra-articularly in human joints. ${ }^{71}$ Pain relieving properties of HA, although not clear, is possibly due to its binding to pain producing peptides and preventing their interaction with pain receptors.

Nevertheless, the evidence for the efficacy of intraarticular HA therapy is contradictory. Three of 5 metaanalysis have reported favorable outcomes, but 2 others did not show significant efficacy following intra-articular HA treatment (Table 1). Analysis of the reasons for the variations in conclusions between the meta-analysis and systemic reviews found differences in search strategies, selection criteria, outcome measures and statistical methods. ${ }^{72}$ The Agency for Healthcare Research and Quality (AHRQ, September 2007, www.ahrq.gov) analyzed the available literature including 42 randomized controlled trials and 6 meta-analyses and concluded that the trials favored viscosupplementation compared to placebo with respect to pain and functional scores.

Intra-articular administration of HA has also been used for osteoarthritis of the hip, ankle and shoulder joints. ${ }^{73-75}$ A pilot study with 13 patients assessed the potential effectiveness of HA therapy in the treatment of lumbar facet joint arthritis, but failed to demonstrate any benefit of the single injection of HA in the management of lumbar facet generated pain. ${ }^{76}$ For the potential application of HA for degenerative IVD, the demonstrated therapeutic role of HA in joint cartilage repair may also be beneficial for disc regeneration because NP and EP cells of the IVD share many common characteristics with chondrocytes in terms of function and extracellular matrix components. Further research is required to explore the application of $\mathrm{HA}$ in this area.

\section{Summary}

The intrinsic biocompatibility of HA and its unique physiochemical properties make it attractive for application in surgery, arthritis treatment, drug delivery and
Table I Clinical trials of HA for the treatment of osteoarthritis of the knee

\begin{tabular}{|c|c|c|}
\hline Author & Included trials & Conclusions \\
\hline Bellamy ${ }^{77}$ & $\begin{array}{l}76 \text { RCT including } \\
40 \text { placebo } \\
\text { controls }\end{array}$ & $\begin{array}{l}\text { Viscosupplementation } \\
\text { is an effective treatment } \\
\text { for OA of the knee with } \\
\text { beneficial effects on pain, } \\
\text { function and patient } \\
\text { global assessment. }\end{array}$ \\
\hline Lo $^{78}$ & $\begin{array}{l}22 \text { placebo } \\
\text { controlled RCT }\end{array}$ & $\begin{array}{l}\text { Intra-articular HA has } \\
\text { a small effect compared } \\
\text { with an intra-articular } \\
\text { placebo. This difference } \\
\text { may be over-estimated } \\
\text { due to publication bias. } \\
\text { Highest-molecular- } \\
\text { weight HA may be more } \\
\text { efficacious, but hetero- } \\
\text { geneity of studies limits } \\
\text { definitive conclusions. }\end{array}$ \\
\hline Arrich $^{79}$ & $22 \mathrm{RCT}$ & $\begin{array}{l}\text { Intra-articular HA has } \\
\text { not been proven clini- } \\
\text { cally effective and may be } \\
\text { associated with a greater } \\
\text { risk of adverse events. }\end{array}$ \\
\hline Modawa $\left.\right|^{80}$ & $\begin{array}{l}\text { II placebo-controlled } \\
\text { RCT that assessed pain } \\
\text { relief at various } \\
\text { time points }\end{array}$ & $\begin{array}{l}\text { Intra-articular HA was } \\
\text { moderately effective in } \\
\text { relieving knee pain in } \\
\text { patients with OA at 5-7 } \\
\text { and 8-10 weeks after } \\
\text { the last injection but } \\
\text { not at } 15-22 \text { weeks. }\end{array}$ \\
\hline Wang ${ }^{81}$ & $\begin{array}{l}20 \text { placebo-controlled } \\
\text { RCT }\end{array}$ & $\begin{array}{l}\text { Confirmed the } \\
\text { therapeutic efficacy and } \\
\text { safety of intra-articular } \\
\text { injection of HA for OA } \\
\text { of the knee. Patients } \\
\text { above } 65 \text { years and } \\
\text { those with advanced } \\
\text { OA less likely to benefit. }\end{array}$ \\
\hline
\end{tabular}

Abbreviations: HA, hyaluronan; OA, osteoarthritis; RCT, randomized controlled trials.

in the emerging area of tissue engineering. HA is not only a promising biomaterial for scaffolds and carriers, but has an array of biological functions. HA may alter function and signaling pathway of stem cells and their responsiveness to growth factors in tissue regeneration approaches since HA-mediated changes in cell-matrix interactions may influence cell responses to signal molecules. The optimal combination of HA with cells and growth factors requires extensive in vitro and in vivo studies. Currently, there is a lack of data on the application of HA in IVD repair. While using stem cells and 
recombinant growth factors for IVD regeneration has attracted great interest, application of HA might speed up the development of disc regeneration strategies by providing an implant with a biologically and mechanically natural microenvironment in the degenerated site.

\section{Disclosures}

The authors declare no conflicts of interest.

\section{References}

1. Meyer K, Palmer J. The polysaccharide of the vitreous humor. J Biol Chem. 1934;107:629-634.

2. Laurent TC, Fraser JR. Hyaluronan. Faseb J. 1992;6(7):2397-2404.

3. Yamada T, Kawasaki T. Microbial synthesis of hyaluronan and chitin: New approaches. J Biosci Bioeng. 2005;99(6):521-528.

4. Balazs EA. Hyaluronan as an ophthalmic viscoelastic device. Curr Pharm Biotechnol. 2008;9(4):236-238.

5. Balazs EA. Viscosupplementation for treatment of osteoarthritis: from initial discovery to current status and results. Surg Technol Int. 2004;12:278-289.

6. Migliore A, Granata M. Intra-articular use of hyaluronic acid in the treatment of osteoarthritis. Clin Interv Aging. 2008;3(2):365-369.

7. Fraser JR, Laurent TC, Laurent UB. Hyaluronan: its nature, distribution, functions and turnover. J Intern Med. 1997;242(1):27-33.

8. Herrera MB, Bussolati B, Bruno S, et al. Exogenous mesenchymal stem cells localize to the kidney by means of CD44 following acute tubular injury. Kidney Int. 2007;72(4):430-441.

9. Park JB, Kwak HJ, Lee SH. Role of hyaluronan in glioma invasion. Cell Adh Migr. 2008;2(3):02-207.

10. Cantor JO. Potential therapeutic applications of hyaluronan in the lung. Int J Chron Obstruct Pulmon Dis. 2007;2(3):283-288.

11. Brunelli G, Longinotti C, Bertazzo C, et al. Adhesion reduction after knee surgery in a rabbit model by Hyaloglide, a hyaluronan derivative gel. J Orthop Res. 2005;23(6):1377-1382.

12. Bastow ER, Byers S, Golub SB, et al. Hyaluronan synthesis and degradation in cartilage and bone. Cell Mol Life Sci. 2008;65(3): 395-413.

13. Volpi N, Schiller J, Stern R, et al. Role, metabolism, chemical modifications and applications of hyaluronan. Curr Med Chem. 2009;16(14):1718-1745.

14. Scott JE, Heatley F. Hyaluronan forms specific stable tertiary structures in aqueous solution: a 13C NMR study. Proc Natl Acad Sci U S A. 1999;96(9):4850-4855.

15. Kobayashi Y, Okamoto A, Nishinari K. Viscoelasticity of hyaluronic acid with different molecular weights. Biorheology. 1994;31(3):235-244.

16. Isacke CM, Yarwood H. The hyaluronan receptor, CD44. Int J Biochem Cell Biol. 2002;34(7):718-721.

17. McCourt PA, Ek B, Forsberg N, et al. Intercellular adhesion molecule-1 is a cell surface receptor for hyaluronan. $J$ Biol Chem. 1994;269(48):30081-30084.

18. Turley EA, Noble PW, Bourguignon LY. Signaling properties of hyaluronan receptors. J Biol Chem. 2002;277(7):4589-4592.

19. Stern R, Asari AA, Sugahara KN. Hyaluronan fragments:an information-rich system. Eur J Cell Bio. 2006;85(8):699-715.

20. Glowacki J, Mizuno S. Collagen scaffolds for tissue engineering. Biopolymers. 2008;89(5):338-344.

21. Allemann F, Mizuno S, Eid K, et al. Effects of hyaluronan on engineered articular cartilage extracellular matrix gene expression in 3-dimensional collagen scaffolds. J Biomed Mater Res. 2001;55(1):13-19.

22. Cavallo C, Desando G, Facchini A, et al. Chondrocytes from patients with osteoarthritis express typical extracellular matrix molecules once grown onto a three-dimensional hyaluronan-based scaffold. J Biomed Mater Res A. 2009;93A(1):86-95.
23. Tan $\mathrm{H}$, Chu CR, Payne KA, et al. Injectable in situ forming biodegradable chitosan-hyaluronic acid based hydrogels for cartilage tissue engineering. Biomaterials. 2009;30(13):2499-2506.

24. Kondo T, Shinozaki T, Oku H, et al. Konjac glucomannan-based hydrogel with hyaluronic acid as a candidate for a novel scaffold for chondrocyte culture. J Tissue Eng Regen Med. 2009;3(5):361-367.

25. Ko CS, Huang JP, Huang CW, et al. Type II collagen-chondroitin sulfate-hyaluronan scaffold cross-linked by genipin for cartilage tissue engineering. J Biosci Bioeng. 2009;107(2):177-182.

26. Chung C, Erickson IE, Mauck RL, et al. Differential behavior of auricular and articular chondrocytes in hyaluronic acid hydrogels. Tissue Eng Part A. 2008;14(7):1121-1131.

27. Goodstone NJ, Cartwright A, Ashton B. Effects of high molecular weight hyaluronan on chondrocytes cultured within a resorbable gelatin sponge. Tissue Eng. 2004;10(3-4):621-631.

28. Kawasaki K, Ochi M, Uchio Y, et al. Hyaluronic acid enhances proliferation and chondroitin sulfate synthesis in cultured chondrocytes embedded in collagen gels. J Cell Physiol. 1999;179(2):142-148.

29. Akmal M, Singh A, Anand A, et al. The effects of hyaluronic acid on articular chondrocytes. J Bone Joint Surg Br. 2005;87(8):1143-1149.

30. Takahashi K, Hashimoto S, Kubo T, et al. Effect of hyaluronan on chondrocyte apoptosis and nitric oxide production in experimentally induced osteoarthritis. J Rheumatol. 2000;27(7):1713-1720.

31. Grishko V, Xu M, Ho R, et al. Effects of hyaluronic acid on mitochondrial function and mitochondria-driven apoptosis following oxidative stress in human chondrocytes. J Biol Chem. 2009;284(14):9132-9139.

32. Hegewald AA, Ringe J, Bartel J, et al. Hyaluronic acid and autologous synovial fluid induce chondrogenic differentiation of equine mesenchymal stem cells: a preliminary study. Tissue Cell. 2004;36(6):431-438.

33. Pasquinelli G, Orrico C, Foroni L, et al. Mesenchymal stem cell interaction with a non-woven hyaluronan-based scaffold suitable for tissue repair. J Anat. 2008;213(5):520-530.

34. Facchini A, Lisignoli G, Cristino S, et al. Human chondrocytes and mesenchymal stem cells grown onto engineered scaffold. Biorheology. 2006;43(3-4):471-480.

35. Zavan B, Giorgi C, Bagnara GP, et al. Osteogenic and chondrogenic differentiation:comparison of human and rat bone marrow mesenchymal stem cells cultured into polymeric scaffolds. Eur J Histochem. 2007; 51 Suppl 1:1-8.

36. Dvorakova J, Velebny V, Kubala L, Hyaluronan influence on the onset of chondrogenic differentiation of mesenchymal stem cells. Neuro Endocrinol Lett. 2008;29(5):685-690.

37. Jha AK, Yang W, Kirn-Safran CB, et al. Perlecan domain I-conjugated, hyaluronic acid-based hydrogel particles for enhanced chondrogenic differentiation via BMP-2 release. Biomaterials. 2009;30(36):6964-6975.

38. Stok KS, Lisignoli G, Cristino S, et al. Mechano-functional assessment of human mesenchymal stem cells grown in three-dimensional hyaluronan-based scaffolds for cartilage tissue engineering. $J$ Biomed Mater Res A. 2009;93A(1):37-45.

39. Garcia-Fuentes M, Meinel AJ, Hilbe M, et al. Silk fibroin/hyaluronan scaffolds for human mesenchymal stem cell culture in tissue engineering. Biomaterials. 2009;30(28):5068-5076.

40. Angele P, Muller R, Schumann D, et al. Characterization of esterified hyaluronan-gelatin polymer composites suitable for chondrogenic differentiation of mesenchymal stem cells. J Biomed Mater Res A. 2009;91(2):416-427.

41. Angele P, Johnstone B, Kujat R, et al. Stem cell based tissue engineering for meniscus repair. J Biomed Mater Res A. 2008;85(2):445-455.

42. Loken S, Jakobsen RB, Aroen A, et al. Bone marrow mesenchymal stem cells in a hyaluronan scaffold for treatment of an osteochondral defect in a rabbit model. Knee Surg Sports Traumatol Arthrosc. 2008;16(10):896-903.

43. Zhang J, Skardal A, Prestwich GD. Engineered extracellular matrices with cleavable crosslinkers for cell expansion and easy cell recovery. Biomaterials. 2008;29(34):4521-4531.

44. Solchaga LA, Temenoff JS, Gao J, et al. Repair of osteochondral defects with hyaluronan- and polyester-based scaffolds. Osteoarthritis Cartilage. 2005;13(4):297-309. 
45. Park SH, Park SR, Chung SI, et al. Tissue-engineered cartilage using fibrin/hyaluronan composite gel and its in vivo implantation. Artif Organs. 2005;29(10):838-845.

46. Hahn SK, Kim SJ, Kim MJ, et al. Characterization and in vivo study of sustained-release formulation of human growth hormone using sodium hyaluronate. Pharm Res. 2004;21(8):1374-1381.

47. Luo Y, Kirker KR, Prestwich GD. Cross-linked hyaluronic acid hydrogel films:new biomaterials for drug delivery. J Control Release. 2000;69(1):169-184.

48. Kim A, Checkla DM, Dehazya P, et al. Characterization of DNAhyaluronan matrix for sustained gene transfer. J Control Release. 2003;90(1):81-95.

49. Segura T, Chung PH, Shea LD. DNA delivery from hyaluronic acidcollagen hydrogels via a substrate-mediated approach. Biomaterials. 2005;26(13):1575-1584.

50. Lee H, Mok H, Lee S, et al. Target-specific intracellular delivery of siRNA using degradable hyaluronic acid nanogels. J Control Release. 2007;119(2):245-252.

51. Jiang G, Park K, Kim J, et al. Target specific intracellular delivery of siRNA/PEI-HA complex by receptor mediated endocytosis. Mol Pharm. 2009;6(3):727-737.

52. Liao E, Yaszemski M, Krebsbach P, et al. Tissue-engineered cartilage constructs using composite hyaluronic acid/collagen I hydrogels and designed poly(propylene fumarate) scaffolds. Tissue Eng. 2007;13(3):537-550.

53. Pereira RC, Scaranari M, Castagnola P, et al. Novel injectable gel (system) as a vehicle for human articular chondrocytes in cartilage tissue regeneration. J Tissue Eng Regen Med. 2009;3(2):97-106.

54. Park SH, Cui JH, Park SR, et al. Potential of fortified fibrin/hyaluronic acid composite gel as a cell delivery vehicle for chondrocytes. Artif Organs. 2009;33(6):439-447.

55. Raj PP. Intervertebral disc: anatomy-physiology-pathophysiologytreatment. Pain Pract. 2008;8(1):18-44.

56. Chung SA, Khan SN, Diwan AD. The molecular basis of intervertebral disk degeneration. Orthop Clin North Am. 2003;34(2):209-219.

57. Yang SH, Chen PQ, Chen YF, et al. An in-vitro study on regeneration of human nucleus pulposus by using gelatin/chondroitin-6-sulfate/hyaluronan tri-copolymer scaffold. Artif Organs. 2005;29(10):806-814.

58. Halloran DO, Grad S, Stoddart M, et al. An injectable cross-linked scaffold for nucleus pulposus regeneration. Biomaterials. 2008;29(4):438-447.

59. Alini M, Li W, Markovic P, et al. The potential and limitations of a cell-seeded collagen/hyaluronan scaffold to engineer an intervertebral disc-like matrix. Spine (Phila Pa 1976). 2003;28(5):446-454; discussion 453.

60. Haberstroh K, Enz A, Zenclussen ML, et al. Human intervertebral disc-derived cells are recruited by human serum and form nucleus pulposus-like tissue upon stimulation with TGF-beta3 or hyaluronan in vitro. Tissue Cell. 2009;41(6):414-420.

61. Schimizzi AL, Massie JB, Murphy M, et al. High-molecular-weight hyaluronan inhibits macrophage proliferation and cytokine release in the early wound of a preclinical postlaminectomy rat model. Spine J. 2006;6(5):550-556.

62. Massie JB, Schimizzi AL, Huang B, et al. Topical high molecular weight hyaluronan reduces radicular pain post laminectomy in a rat model. Spine J. 2005;5(5):494-502.
63. Nesti LJ, Li WJ, Shanti RM, et al. Intervertebral disc tissue engineering using a novel hyaluronic acid-nanofibrous scaffold (HANFS) amalgam. Tissue Eng Part A. 2008;14(9):1527-1537.

64. Crevensten G, Walsh AJ, Ananthakrishnan D, et al. Intervertebral disc cell therapy for regeneration:mesenchymal stem cell implantation in rat intervertebral discs. Ann Biomed Eng. 2004;32(3):430-434.

65. Revell PA, Damien E, Di Silvio L, et al. Tissue engineered intervertebral disc repair in the pig using injectable polymers. JMater Sci Mater Med, 2007;18(2):303-308.

66. Krasnokutsky S, Attur M, Palmer G, et al. Current concepts in the pathogenesis of osteoarthritis. Osteoarthritis Cartilage. 2008;16 Supp1 3:S1-S3.

67. Watterson JR, Esdaile JM. Viscosupplementation:therapeutic mechanisms and clinical potential in osteoarthritis of the knee. J Am Acad Orthop Surg. 2000;8(5):277-284.

68. Ling PX, Rong XH, Zhang TM. Application of sodium hyaluronate in surgery. Hyaluronan: Structure, Metabolism, Biological Activities, Therapeutic Applications. 2005;2:509-511.

69. Gomis A, Pawlak M, Balazs EA, et al. Effects of different molecular weight elastoviscous hyaluronan solutions on articular nociceptive afferents. Arthritis Rheum. 2004;50(1):314-326.

70. Strauss E, Schachter A, Frenkel S, et al. The efficacy of intra-articular hyaluronan injection after the microfracture technique for the treatment of articular cartilage lesions. Am J Sports Med. 2009;37(4):720-726.

71. Balazs EA. Analgesic effect of elastoviscous hyaluronan solutions and the treatment of arthritic pain. Cells Tissues Organs. 2003;174(1-2):49-62.

72. Campbell J, Bellamy N, Gee T. Differences between systematic reviews/ meta-analyses of hyaluronic acid/hyaluronan/hylan in osteoarthritis of the knee. Osteoarthritis Cartilage. 2007;15(12):1424-1436.

73. Diracoglu D, Alptekin K, Teksoz B, et al. Knee vs hip single-joint intraarticular hyaluronic acid injection in patients with both hip and knee osteoarthritis: a pilot study. Clin Rheumatol. 2009;28(9):1021-1024.

74. Luciani D, Cadossi M, Tesei F, et al. Viscosupplementation for grade II osteoarthritis of the ankle:a prospective study at 18 months' follow-up. Chir Organi Mov. 2008;92(3):155-160.

75. Blaine T, Moskowitz R, Udell J, et al. Treatment of persistent shoulder pain with sodium hyaluronate: a randomized, controlled trial. A multicenter study. J Bone Joint Surg Am . 2008;90(5):970-979.

76. Cleary M, Keating C, Poynton AR. Viscosupplementation in lumbar facet joint arthropathy: a pilot study. J Spinal Disord Tech. 2008;21(1):29-32.

77. Bellamy N, Campbell J, Robinson V, et al. Viscosupplementation for the treatment of osteoarthritis of the knee. Cochrane Database Syst Rev. 2006(2):CD005321.

78. Lo GH, LaValley M, McAlindon $\mathrm{T}$, et al. Intra-articular hyaluronic acid in treatment of knee osteoarthritis:a meta-analysis. JAMA. 2003;290(23):3115-3121.

79. Arrich J, Piribauer F, Mad P, et al. Intra-articular hyaluronic acid for the treatment of osteoarthritis of the knee:systematic review and metaanalysis. CMAJ. 2005;172(8):1039-1043.

80. Modawal A, Ferrer M, Choi HK, et al. Hyaluronic acid injections relieve knee pain. J Fam Pract. 2005;54(9):758-767.

81. Wang CT, Lin J, Chang CJ, et al. Therapeutic effects of hyaluronic acid on osteoarthritis of the knee. A meta-analysis of randomized controlled trials. J Bone Joint Surg Am. 2004;86-A(3):538-545.
Orthopedic Research and Reviews

\section{Publish your work in this journal}

Orthopedic Research and Reviews is an international, peer-reviewed, open access journal that focusing on the patho-physiology of the musculoskeletal system, trauma, surgery and other corrective interventions to restore mobility and function. Advances in new technologies, materials, techniques and pharmacological agents are particularly welcome. The journal welcomes Submit your manuscript here: http://www.dovepress.com/orthopedic-research-and-reviews-journa

\section{Dovepress}

original research, clinical studies, reviews \& evaluations, expert opinion and commentary, case reports and extended reports. The manuscript management system is completely online and includes a very quick and fair peer-review system, which is all easy to use. Visit http://www.dovepress. com/testimonials.php to read real quotes from published authors. 